\title{
Implantes unitários curtos e extra-curtos na maxila como alternativa para enxertos ósseos: revisão de literatura e relato de casos clínicos com acompanhamento de 36 a 50 meses
}

\author{
Short and extra-short unitary implants in the maxilla as an \\ alternative for bone grafts: literature review and clinical case \\ reports with follow-up from 36 to 50 months
}

\section{Implantes unitarios cortos y extracortos en el maxilar como alternativa a los injertos óseos: revisión de la literatura e informes de casos clínicos con seguimiento de 36 a 50 meses}

Fabio Jose Barbosa Bezerra (iD

Bruna Ghiraldini iD

Jackson Both

Alexandre Marcelo Carvalho

Lucas Lactim Ferrarez iD

Bruno Salles Sotto Maior iD

1 both@win.com.br

Endereço para correspondência:

Bruno Salles Sotto Maior

Universidade Federal de Juiz de Fora

Rua José Lourenço Kelmer, s/n - Campus Universitário

Bairro São Pedro

36036-900 - Juiz de Fora - Minas Gerais - Brasil

E-mail: brunosottomaior@gmail.com

Recebido: 14.08 .2020

Aceito: 01.09.2020

\section{RESUMO}

Este trabalho teve como objetivo revisar a literatura e relatar dois casos clínicos realizados na região posterior da maxila com alturas ósseas limitadas utilizando implantes unitário curtos e extra-curtos como alternativas às cirurgias de enxerto para elevação do assoalho do seio maxilar e acompanhados por um período de 36 a 50 meses. O planejamento reabilitador contou com a instalação de implantes com macrogeometria híbrida, câmaras de cicatrização e superfície nanoativada para compensar a limitação de altura e baixa densidade óssea presentes na região posterior da maxila. As reabilitações protéticas unitárias foram realizadas através de carga precoce ( 90 dias após a instalação dos implantes) e não houve intercorrências cirúrgicas ou protéticas durante o período de acompanhamento. Dentro dos limites deste estudo pode-se concluir que os implantes curtos ou extra-curtos utilizados em casos unitários podem ser uma alternativa de tratamento mais simples, com menor morbidade cirúrgica e menor tempo global de tratamento quando comparados aos tratamentos realizados com reconstruções ósseas. 
PALAVRAS-CHAVE: Implantes dentários. Osseointegração. Regeneração óssea.

\begin{abstract}
This study aimed to review the literature and report two clinical cases performed in the posterior region of the maxilla with limited bone heights using short and extra-short unitary implants as alternatives to graft surgeries to elevate the maxillary sinus floor and accompanied by a period of 36 to 50 months. The rehabilitation plan included the installation of implants with hybrid macrogeometry, healing chamber and nanoactivated surface to compensate for the height limitation and low bone density present in the posterior region of the maxilla. Unit prosthetic rehabilitations were transmitted through early loading (90 days after implant placement) and there were no surgical or prosthetic complications during the follow-up period. Within the study limits, it can be observed that short or extra-short implants used in single cases can be a simpler treatment alternative, with less surgical morbidity and shorter overall treatment time when compared to treatments performed with bone reconstructions.
\end{abstract}

KEYWORDS: Dental implants. Osseointegration. Bone regeneration.

\title{
RESUMEN
}

Este estudio tuvo como objetivo revisar la literatura y reportar dos casos clínicos realizados en la región posterior del maxilar con alturas óseas limitadas utilizando implantes unitarios cortos y extracortos como alternativas a las cirugías de injerto para elevar el piso del seno maxilar y seguidas por un período de 36 a 50 meses. El plan de rehabilitación incluyó la instalación de implantes con macrogeometría híbrida, cámaras de cicatrización y superficie nanoactivada para compensar la limitación de altura y la baja densidad ósea presente en la región posterior del maxilar. Las rehabilitaciones protésicas unitarias se realizaron mediante carga precoz (90 días después de la instalación del implante) y no hubo complicaciones quirúrgicas ni protésicas durante el período de seguimiento. Dentro de los límites de este estudio, se puede concluir que los implantes cortos o extracortos utilizados en casos únicos pueden ser una alternativa de tratamiento más sencilla, con menor morbilidad quirúrgica y menor tiempo de tratamiento global en comparación con los tratamientos realizados con reconstrucciones óseas.

PALABRAS CLAVE: Implantes dentales. Oseointegración. Regeneración ósea. 


\section{INTRODUÇÃO}

A atrofia óssea frequentemente impõe um desafio no tratamento com implantes dentários, porque a reabsorção óssea vertical e horizontal da crista óssea alveolar e/ou a penumatização do seio maxilar, após a perda dos dentes, podem impedir a colocação de implantes de tamanho padrão ${ }^{1}$.

A limitação anatômica posterior determinada pela reabsorção mandibular relaciona-se ao canal alveolar inferior e na maxila pela pneumatização do seio maxilar exigem técnicas reabilitadoras avançadas para a estabilização do implante ossoeointegrável, como: enxertias para aumento da disponibilidade ósseas e permitir a colocação de implantes longos, distração osteogênica, lateralização do nervo mandibular ${ }^{2}$, e levantamento da membrana do seio maxilar, associado a preenchimento com enxertos ósseos de origem autógena ou biomateriais ${ }^{3.4}$. No entanto tais procedimentos vêm se mostrando dispendiosos e demorados, com elevada morbidade cirúrgica ${ }^{5-8}$. Em casos de elevação do seio maxilares complicações como infecção do seio maxilar, edema, sangramento, sinusites e a perfuração da membrana de Schneider, considerado a principal complicação trans-operatôria representando $23.5 \%$ podem estar associadoas. A perfuração da membrana de Schneider reduz a formação óssea ${ }^{9}$ e em estudo clinico retrospectivo demonstrou-se que a perfuração da membrana aumenta em 3 vezes o risco de falha do enxerto ósseo e 6 vezes a incidência de sinusite e infecção do seio maxilar ${ }^{10}$.

Nesse cenário, implantes curtos, de 6 a $8.5 \mathrm{~mm}$ de comprimentos, e extracurtos, menores de $6 \mathrm{~mm} 11$ proveem uma alternativa na implantodontia para pacientes com rebordos posteriores atróficos ${ }^{12-13}$, principalmente após a evolução dos tratamentos de superfície ${ }^{14} \mathrm{e}$ melhorias em sua macrogeometria ${ }^{14-15}$. Diversos estudos recentes demonstram que os implantes extracurtos têm alcançado taxas de sobrevida e remodelação óssea semelhantes aos implantes longos ${ }^{7,13,16}$. Atualmente, os implantes curtos e extracurtos podem ser considerados uma alternativa previsível à associação de cirurgias reconstrutivas e implantes longos ${ }^{7,16}$.

\section{RELATO DE CASOS}

Foram selecionados dois pacientes com limitação óssea maxilar posterior associada a pneumatização do seio maxilar inviabilizando a instalação de implantes com comprimentos convencionais. As duas pacientes selecionadas são do gênero feminino com 59 (I.G.) e 47 anos (L.A.F.), sem uso de medicação sistêmica, sem alterações metabólicas e não-fumantes, com queixas similares de ausência dentária.
Foram realizadas anamneses sobre o estado geral de saúde das pacientes e exames clínicos com sondagem dos dentes adjacentes, avaliação da estabilidade oclusal, moldagens para confecção de modelos de estudo e protocolo fotográfico padronizado. Ao final da primeira consulta e solicitado exames complementares de imagens, radiografias panorâmicas e periapicais e exames sanguíneos.

Após a avaliação clínica e avaliação dos exames complementares (Figura 1) ficou constado a disponibilidade óssea reduzida de $7 \mathrm{~mm}$ nas duas pacientes devido à ausência dentária e pneumatização dos seios maxilares, portanto foi proposto a instalação de implantes curtos e extra-curtos com posterior reabilitação unitária implanto-suportada. As pacientes receberam protocolo medicamentoso pré-operatório de $2 \mathrm{~g}$ amoxicilina, $4 \mathrm{mg}$ dexametasona uma hora antes do procedimento cirúrgico e bochecho com gluconato de clorexidina a $0.12 \%$ por 1 minuto no pré-operatório imediato.
CASO I.G.

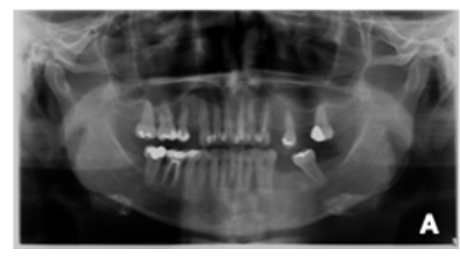

CASO L.A.F.

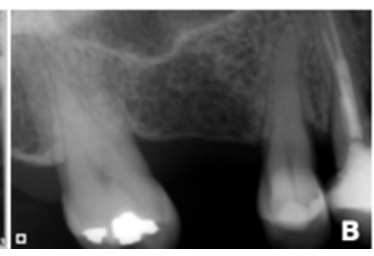

Figura 1 - Imagens radiográficas pré-operatórias dos dois casos apresentados (A) caso clínico I.G. e (B) caso clinico L.A.F.

Foram anestesiados os nervos alveolares superiores médio e posterior e palatino maior com mepivacaina $3 \%$ associado a técnica infiltrativa terminal no local da cirurgia com intuito de hemostasia. Posteriormente foi realizada incisão linear sobre a crista do rebordo alveolar e descolamento de retalho total para a exposição óssea (Figura 2).
CASO I.G.

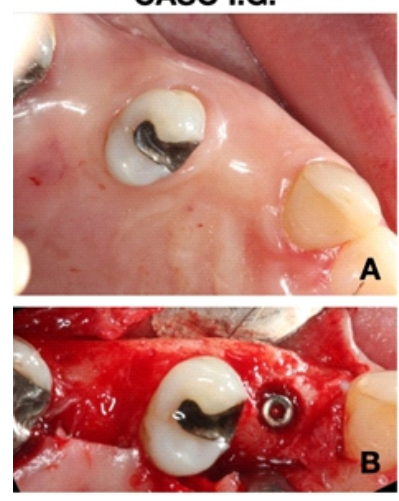

CASO L.A.F.
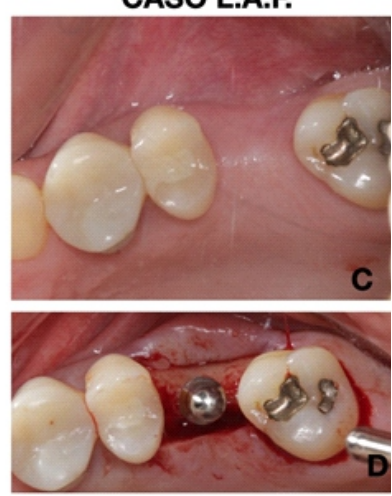

Figura 2 - Imagem clínica pré-operatória e após a incisão linear.

Para os casos selecionados foram escolhidos os implantes Unitite Compact (S.I.N. Implant System, São Paulo, Brasil) de $6 \mathrm{~mm}$ de diâmetro e $7 \mathrm{~mm}$ de comprimento para o caso I.G. e o implante Unitite Compact (S.I.N. 
Implant System, São Paulo, Brasil) de 4 mm de diâmetro e 5 $\mathrm{mm}$ de comprimento para o caso L.A.F., seguindo o protocolo de fresagem preconizado pelo fabricante. Como alternativa de segurança contra eventual perfuração da membrana de Shneider foi utilizado um dispositivo limitador de profundidade, Safe Drill (S.I.N. Implant System, São Paulo, Brasil) (Figura 3).

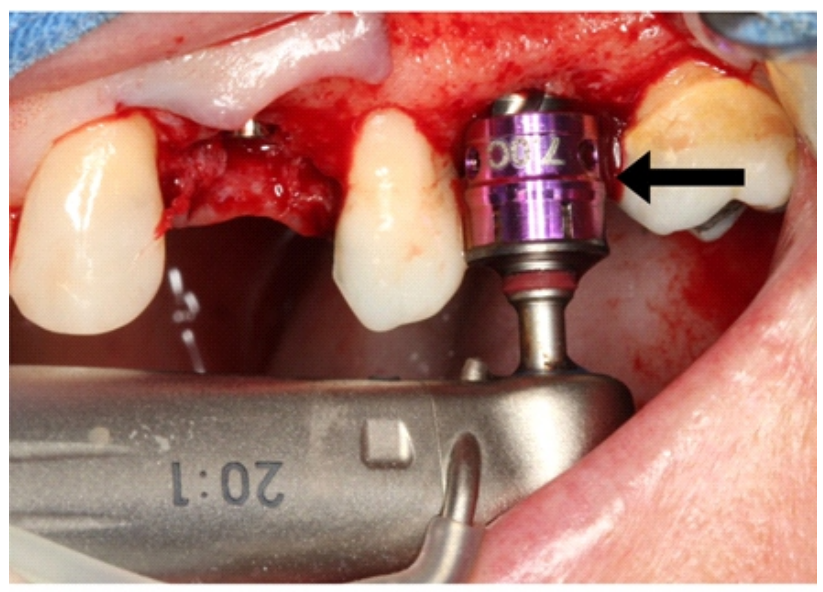

Figura 3 - Imagem do Safe Drill (S.I.N. Implant System, São Paulo, Brasil) destacado com a seta.

Após a instalação dos implantes foram conferidos a estabilidade primária, sendo alcançado $10 \mathrm{Ncm}$ para o caso da paciente I.G. e $30 \mathrm{Ncm}$ para o caso da paciente L.A.F. (Figura 4), portanto sendo instalados os tapas implantes e realizadas suturas simples com fio de nylon 5-0 (Techsuture, São Paulo, Brasil).
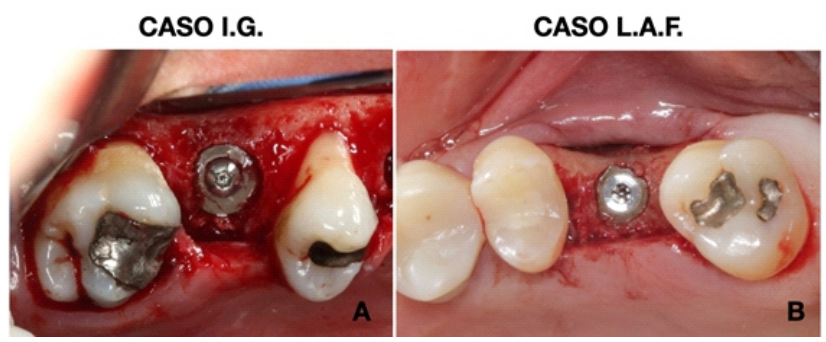

Figura 4 - Imagem clínica dos implantes instalados.

Após o período de 90 dias de osseointegração realizouse a segunda intervenção cirúrgica para instalação de um cicatrizador e após 15 dias, os pilares protéticos para próteses unitárias cimentadas (Abutment Universal, S.I.N. Implant System, São Paulo, Brasil) receberam coroas provisórias para condicionar o perfil de emergência gengival. Seguido da moldagem de transferência com silicone de adição e confeccionadas próteses finais metalocerâmicas.
CASO I.G.

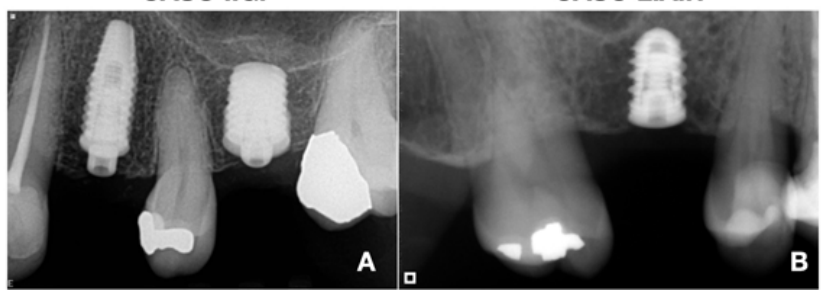

Figura 5 - Radiografias dos implantes posicionados.

Os acompanhamentos radiográficos e clínicos foram realizados no pós-operatório imediato, segunda intervenção cirúrgica (reabertura), imediatamente após a instalação da coroa definitiva (Figura 6), e sequencialmente 12 meses (Figura 7), 24 meses (Figura 8), 34 meses (Figura 9) e 50 meses (Figura 10) após a instalação da coroa para o caso I.G. e 12 meses, 24 meses, 36 meses para o caso L.A.F.

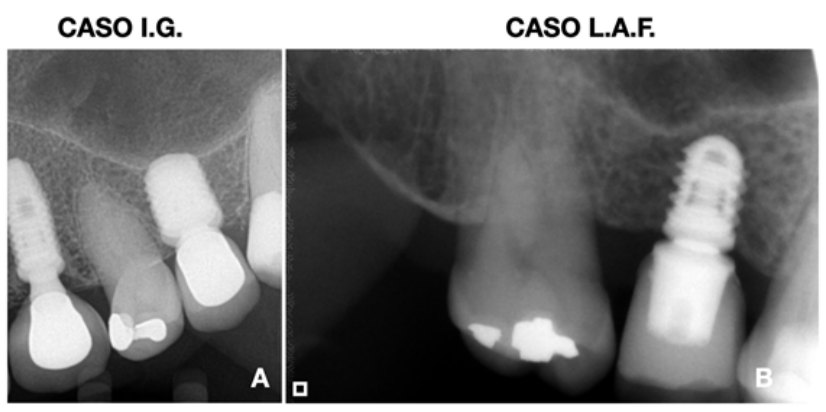

Figura 6 - Imagens radiográficas imediatamente após a instalação da coroa definitiva.

\section{CASO I.G.}

CASO L.A.F.

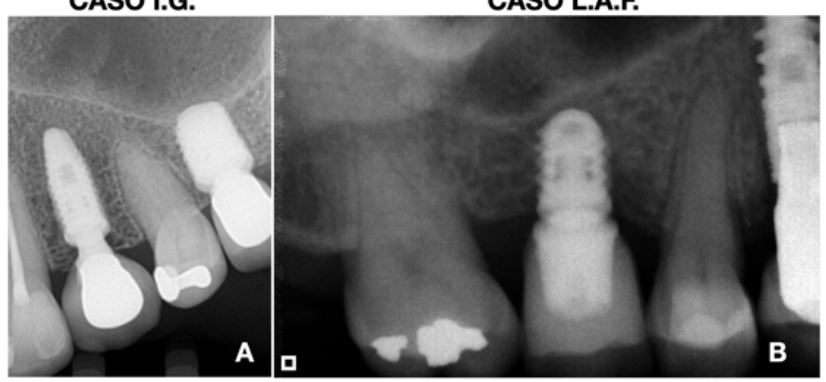

Figura 7 - Imagens radiográficas 12 meses após a instalação da coroa definitiva.
CASO I.G.

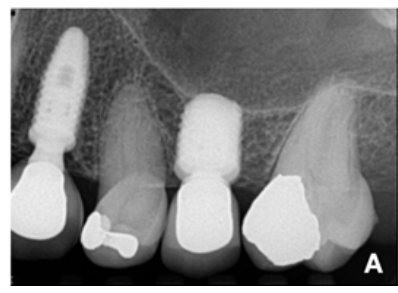

CASO L.A.F.

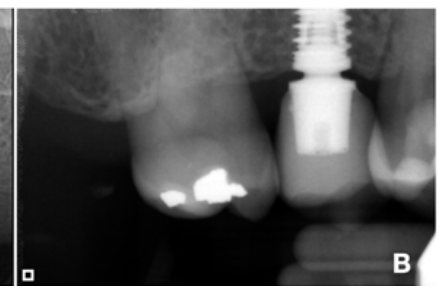

Figura 8 - Imagens radiográficas 24 meses após a instalação da coroa definitiva. 
CASO I.G.

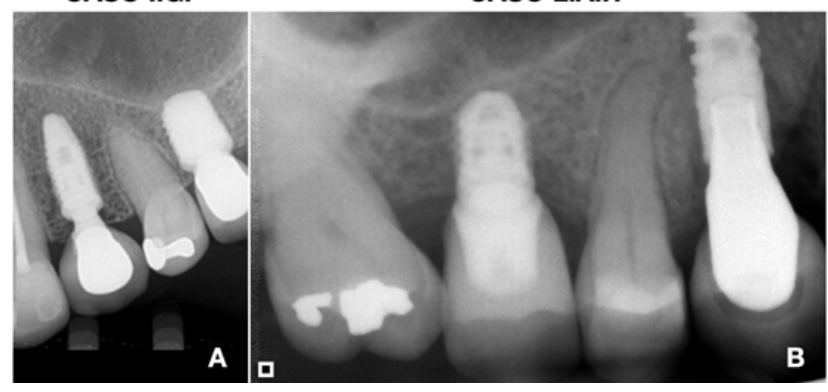

Figura 9 - Imagens radiográficas 36 meses após a instalação da coroa definitiva.

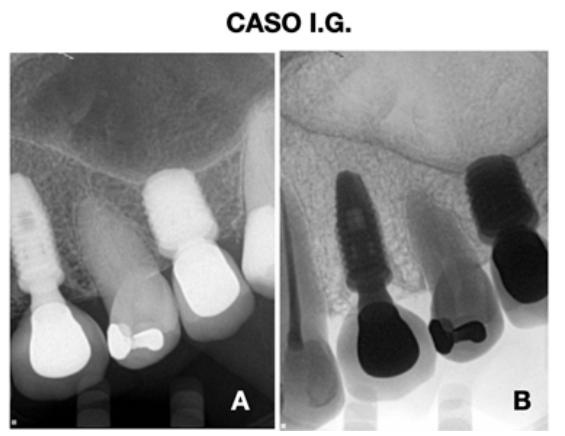

Figura 10 - Imagens radiográficas 50 meses após a instalação da coroa definitiva.

\section{DISCUSSÃO}

O uso de implantes dentários é considerado um procedimento de terapia protética estética e funcional bemsucedida para reabilitar a ausência de elementos dentários ${ }^{17-}$ 18. A ausência de dentes associados a fatores sistêmicos e longos períodos de edentulismo podem resultar em reabsorções na altura e espessura do osso alveolar ${ }^{13}$. A redução da altura pode ser considerada um fator de risco para o tratamento restaurador com implantes dentários, principalmente na região posterior da mandíbula e maxila, onde estruturas anatômicas como o nervo alveolar inferior e o seio maxilar estão presentes ${ }^{19-21}$. Esse fator de risco pode ser potencializados devido às maiores forças mastigatórias $^{22}$ nessas regiões, especialmente em maxilas que apresentam uma densidade óssea reduzida, sendo comumente classificada como osso tipo III ou IV segundo a classificação de Lekholm and Zarb ${ }^{23}$.

A quantidade óssea na região posterior da maxila foi categorizada em três grupos: na classe 1, a altura óssea residual é de pelo menos $10 \mathrm{~mm}$; na classe 2, a altura óssea residual é de 5-10 mm; e na classe 3 , a altura óssea é de 0 - 5 mm24. Nos casos selecionados para esse artigo estão classificados como classe 3. Na maxila, classe 2 ou 3, uma das alternativas a limitação é o procedimento de elevação da membrana do seio maxilar, membrana de Schneider, associado a preenchimento com enxerto ósseo que pode ser de origem autógena, biomaterial ou uma associação entre os dois materiais. Entretanto, as técnicas de enxertia óssea estão associadas ao aumento da morbidade pós-operatória, tempo de tratamento e complicações como perfuração ou dilaceração da membrana de Schneider, sinusites e infecções do seio maxilar ${ }^{25}$.

Como alternativa os implantes curtos e/ou extra-curtos têm sido propostos como opção tratamento para rebordos com limitação disponibilidade óssea em altura para evitando traumas estruturas vitais nobres, minimizando trauma e diminuindo a morbidade e tempo de tratamento de cirurgias cirúrgicas avançadas ${ }^{1,26}$. No presente estudo foram apresentados dois casos clínicos com essa alternativa terapêutica com acompanhamento clínico e radiográfico de longo prazo, 36 e 50 meses em concordância com vários estudos clínicos ${ }^{1,10,13,20-21}$ que concluem similaridade nas taxas de sucessos dos implantes curtos e extra-curtos em comparação com implantes convencionais. Em estudo clinico avaliou-se 77 implantes curtos ou implantes extracurtos e 69 implantes convencionas instalados em mandíbula e maxila com carregamento imediato e acompanhamento por até 4 anos constando taxas de sucessos de similares entre os comprimentos dos implantes avaliados, $99.3 \%$ para os implantes convencionais e $98.4 \%$ para os implantes curtos e extra-curtos ${ }^{1}$.

Em estudo biomecânico com a metodologia de elementos finitos demonstrou-se que as tensões provenientes da forca mastigatória concentram nas primeiras roscas do implante independente do seu comprimento. Dessa forma o aumento do diâmetro do implante, especialmente nos implantes extra-curtos e curtos devem ser preferíveis sempre que possível. Com esse conceito nos casos selecionados forma utilizados implantes de plataforma larga, 6 mm e 4 mm de diâmetro ${ }^{27}$.

$\mathrm{Na}$ maxila posterior, os implantes, especialmente os curtos e extra-curtos, estão mais sujeitos a estabilidade primária reduzida devido a característica biomecânica desfavorável na interface osso-implante, classificada como osso tipo III ou IV segundo a classificação de Lekholm and $Z_{\text {arb }}{ }^{28}$. Dificuldade essa na estabilidade primária observado nos casos realizados que podemos considerar baixa estabilidade alcançada. Para superar essa dificuldade favorecendo a estabilidade primária deve-se preferencialmente utilizar implantes mais largos, utilizar técnicas cirúrgicas que favoreçam a estabilidade primária como subfresagem e osseodensificação.

Em regiões de osso tipo IV de Lekholm and Zarb, também, apresentam um desafio maior na ossoeintegração como demonstrado por vários estudos ${ }^{29-31}$ com taxas menores quando comparados a osso tipo II e III. Portanto, é indicado a utilização de implantes com tratamento de superfície bioativa e alta hidrofilia que favorecem o reparo 
ósseo. Estudos ${ }^{32-33}$ in vitro mostraram que a topografia da superfície do implante tem um efeito importante na proliferação e diferenciação de osteoblastos. O aumento da atividade de osteoblastos pode ser visto com superfícies de implante moderadamente rugosas (rugosidade de superfície de 1 a $2 \mu \mathrm{m}$ ) em comparação com superfícies lisas ou não tratadas ${ }^{34-35}$, além de apresentar um aumento na área de superfície do implante até aproximadamente seis vezes36. Frente a esse desafio adotamos o uso de implantes com tratamento de superfície com nano partícula hidroxiapatita HA com espessura homogênea de $20 \mathrm{~mm}$ sobre uma superfície de titânio grau 4 com textura moderadamente rugosa. Este aspecto é altamente desejável quando as condições desafiadoras para a osseointegração, como regiões de osso tipo III e/ou IV.

\section{CONCLUSÃO}

Dentro dos limites deste estudo, os casos apresentados demonstram a viabilidade clínica da utilização dos implantes curtos e extra-curtos para a reabilitação oral unitária em maxilas com pneumatização do seio maxilar.

\section{REFERÊNCIAS}

1. Amato F, Polara G, Spedicato GA. immediate loading of fixed partial dental prostheses on extra-short and short implants in patients with severe atrophy of the posterior maxilla or mandible: an up-to-4-year clinical study. Int J Oral Maxillofac Implants. 2020;35(3):607-15.

2. Campos CG, Francischone CE, Souza Picorelli Assis NM, Devito $\mathrm{KL}$, Sotto-Maior BS. neurosensory function and implant survival rate following implant placement with or without an interposed bone graft between the implant and nerve: prospective clinical trial. Int J Oral Maxillofac Implants. 2019;33(6):1450-6.

3. Sotto-Maior BS, Senna PM, da Silva WJ, Rocha EP, Del Bel Cury AA. Influence of crown-to-implant ratio, retention system, restorative material, and occlusal loading on stress concentrations in single short implants. Int J Oral Maxillofac Implants. 2012;27(3):e13-8.

4. Sotto-Maior BS, Senna PM, Silva-Neto JP, de Arruda Nóbilo MA, Cury AA. Influence of crown-to-implant ratio on stress around single short-wide implants: a photoelastic stress analysis. J Prosthodont. 2015;24(1):52-6.

5. Esposito M. Short dental implants and non-implant-related articles? Yes, we can! Eur J Oral Implantol. 2009;2(1):3.

6. Esposito M, Zucchelli G, Barausse C, Pistilli R, Trullenque-Eriksson A, Felice P. Four mm-long versus longer implants in augmented bone in atrophic posterior jaws: 4-month post-loading results from a multicentre randomised controlled trial. Eur J Oral Implantol. 2016;9(4):393-409.
7. Felice P, Barausse C, Pistilli R, Ippolito DR, Esposito M. Five-year results from a randomised controlled trial comparing prostheses supported by 5 -mm long implants or by longer implants in augmented bone in posterior atrophic edentulous jaws. Int J Oral Implantol (Berl). 2019;12(1):25-37.

8. Villarinho EA, Triches DF, Alonso FR, Mezzomo LAM, Teixeira ER, Shinkai RSA. Risk factors for single crowns supported by short (6$\mathrm{mm}$ ) implants in the posterior region: A prospective clinical and radiographic study. Clin Implant Dent Relat Res. 2017;19(4):671-80.

9. Proussaefs P, Lozada J, Kim J, Rohrer MD. Repair of the perforated sinus membrane with a resorbable collagen membrane: a human study. Int J Oral Maxillofac Implants. 2004;19(3):413-20.

10. Nolan PJ, Freeman K, Kraut RA. Correlation between Schneiderian membrane perforation and sinus lift graft outcome: a retrospective evaluation of 359 augmented sinus. J Oral Maxillofac Surg. 2014;72(1):47-52.

11. Al-Johany SS, Al Amri MD, Alsaeed S, Alalola B. Dental implant length and diameter: a proposed classification scheme. J Prosthodont. 2017;26(3):252-60.

12. Mendonca JA, Francischone CE, Senna PM, de Oliveira AEM, Sotto-Maior BS. A retrospective evaluation of the survival rates of splinted and non-splinted short dental implants in posterior partially edentulous jaws. J Periodontol. 2014;85(6):787-94.

13. Anitua E, Piñas L, Begoña L, Orive G. Long-term retrospective evaluation of short implants in the posterior areas: clinical results after 10-12 years. J Clin Periodontol. 2014;41(4):404-11.

14. Fabris V, Manfro R, Reginato VF, Bacchi A. Rehabilitation of a severely resorbed posterior mandible with 4-mm extra-short implants and guided bone regeneration: case report with 3-year follow-up. Int J Oral Maxillofac Implants. 2018;33(5):e147-50.

15. Lombardo G, Marincola M, Signoriello A, Corrocher G, Nocini PF. Single-crown, short and ultra-short implants, in association with simultaneous internal sinus lift in the atrophic posterior maxilla: a three-year retrospective study. Materials (Basel). 2020;13(9).

16. Esposito M, Buti J, Barausse C, Gasparro R, Sammartino G, Felice P. Short implants versus longer implants in vertically augmented atrophic mandibles: A systematic review of randomised controlled trials with a 5-year post-loading follow-up. Int J Oral Implantol (Berl). 2019;12(3):267-80.

17. Markovi? A, ?oli? S, Š?epanovi? M, Miši? T, Đini? A, Bhusal DS. A 1 -year prospective clinical and radiographic study of early-loaded bone level implants in the posterior maxilla. Clin Implant Dent Relat Res. 2015;17(5):1004-13.

18. Zembic A, Bosch A, Jung RE, Hammerle CH, Sailer I. Five-year results of a randomized controlled clinical trial comparing zirconia and titanium abutments supporting single-implant crowns in canine and posterior regions. Clin Oral Implants Res. 2013;24(4):384-90.

19. Misch CE. Short dental implants: a literature review and rationale for use. Dent Today. 2005;24(8):64-6,68.

20. Rossi F, Ricci E, Marchetti C, Lang NP, Botticelli D. Early loading of single crowns supported by $6-\mathrm{mm}$-long implants with a moderately rough surface: a prospective 2-year follow-up cohort study. Clin Oral Implants Res. 2010;21(9):937-43.

21 Rossi F, Botticelli D, Cesaretti G, De Santis E, Storelli S, Lang NP. Use of short implants $(6 \mathrm{~mm})$ in a single-tooth replacement: a 5-year follow-up prospective randomized controlled multicenter clinical 
Implantes unitários curtos e extra-curtos na maxila como alternativa para enxertos ósseos: revisão de literatura e relato de casos clínicos com acompanhamento de 36 a 50 meses

study. Clin Oral Implants Res. 2016;27(4):458-64.

22. Koc D, Dogan A, Bek B. Bite force and influential factors on bite force measurements: a literature review. Eur J Dent. 2010;4(2):223-32.

23. Linck GK, Ferreira GM, De Oliveira RC, Lindh C, Leles CR, Ribeiro-Rotta RF. The influence of tactile perception on classification of bone tissue at dental implant insertion. Clin Implant Dent Relat Res. 2016;18(3):601-8.

24. Nimigean V, Nimigean VR, M?ru N, S?1?v?stru DI, B?di?? D, Tuculin? MJ. The maxillary sinus floor in the oral implantology. Rom J Morphol Embryol. 2008;49(4):485-9.

25. Esfahrood ZR, Ahmadi L, Karami E, Asghari S. Short dental implants in the posterior maxilla: a review of the literature. J Korean Assoc Oral Maxillofac Surg. 2017;43(2):70-6.

26. Xu X. Short implants versus longer implants in the posterior alveolar region after an observation period of at least five years: a systematic review and meta-analysis. J Dent. 2020;100:103386.

27. Anitua E, Tapia R, Luzuriaga F, Orive G. Influence of implant length, diameter, and geometry on stress distribution: a finite element analysis. Int J Periodontics Restor Dent. 2010;30(1):89-95.

28. Renouard F, Nisand D. Short implants in the severely resorbed maxilla: A 2-year retrospective clinical study. Clinl Implant Dent Relat Resh. 2005;7:S104-10.

29. Chang LC. Risk factors associated with early failure of maxillary versus mandibular implants: a retrospective study. Int J Oral Implantol(Berl). 2020;13(1):55-63.

30. Martinolli M. Long-term survival analysis of standard-length and short implants with multifunctional abutments. J Oral Rehabil. 2019;46(7):640-6.

31. Pommer B, Mailath-Pokorny G, Haas R, Buseniechner D, Millesi W, Fürhauser R. Extra-short $(<7 \mathrm{~mm})$ and extra-narrow diameter $(<3.5$ $\mathrm{mm}$ ) implants: a meta-analytic literature review. Eur J Oral Implantol. 2018;11(Suppl 1):S137-46.

32. Cecchinato F, Atefyekta S, Wennerberg A, Andersson M, Jimbo R, Davies JR. Modulation of the nanometer pore size improves magnesium adsorption into mesoporous titania coatings and promotes bone morphogenic protein 4 expression in adhering osteoblasts. Dent Mater. 2016;32(7):e148-58.

33. Marinucci L. Effect of titanium surface roughness on human osteoblast proliferation and gene expression in vitro. Int $\mathrm{J}$ Oral Maxillofac Implants. 2006;21(5):719-25.

34. Albrektsson T, Wennerberg A. On osseointegration in relation to implant surfaces. Clin Implant Dent Relat Res. 2019;21(Suppl 1):4-7.

35. Wennerberg A, Albrektsson T, Chrcanovic B. Long-term clinical outcome of implants with different surface modifications. Eur J Oral Implantol. 2018;11(Suppl 1):S123-36.

36. von Wilmowsky C, Moest T, Nkenke E, Stelzle F, Schlegel KA. Implants in bone: part I. A current overview about tissue response, surface modifications and future perspectives. Oral Maxillofac Surg. 2014;18(3):243-57. 